\section{Anemia ferropriva em escolares de Campinas, São Paulo: prevalência, sensibilidade e especificidade de testes laboratoriais}

\author{
Iron deficiency anemia among \\ schoolchildren from Campinas, Sao Paulo: \\ prevalence, sensitivity and specificity of \\ laboratory tests
}

Erly Catarina de Moura 1

Ana Magaly Santos 2

Cláudio Evair Pacheco 3

1-3 Faculdade de Ciências Médicas da Pontíficia Universidade Católica de Campinas, São Paulo.

Avenida John Boyd Dunlop s/n. Campinas, São Paulo, Brasil, CEP $13.059-900$

\begin{abstract}
Objectives: to identify the prevalence of iron deficiency anemia and to calculate the sensitivity and specificity of laboratory tests, specifically hemoglobin and hematocrit, to predict an iron deficiency, considering serum ferritin as the standard test.

Methods: the study involved 365 schoolchildren from Campinas, Sao Paulo,Brazil aged from 7 to 14 years old. The children were considered to have anemia when they presented hemoglobin concentration under 11,5 $\mathrm{g} /$ dl for children aged $5 \leq 8$ years old, $11,9 \mathrm{~g} / \mathrm{dl}$ or children aged $8 \leq 12$ years old, $12,5 \mathrm{~g} / \mathrm{dl}$ for males and 11,8g/dl for females aged $12 \leq 15$ years old. For hematocrit the children were considered to have anemia when the values were under $34,5 \%$, $35,4 \%, 37,3 \%$ and $35,7 \%$, for the same strata. For serum ferritin, under $15 \mu \mathrm{g} / \mathrm{dl}$.

Results: the results showed anemia in 12,4\% of the studied population according to hemoglobin concentration, 7,5\% according to hematocrit level and $19,3 \%$ according to serum ferritin. The prevalence of iron deficiency anemia was 19,3\% according to the ferritin standard. The sensitivity and specificity of hemoglobin concentration to predict anemia was $12,9 \%$ and $87,7 \%$, respectively and the sensitivity and specificity of hematocrit were $8,6 \%$ and $92,8 \%$.

Conclusions: these data indicate the failure of hemoglobin and hematocrit tests to prognose iron deficiency anemia. It is necessary to review the diagnosis procedure in order to improve health assistance, es pecially in the primary health services of Brazil.
\end{abstract}

Key words Anemia iron deficciency, Ferritin, School health, Public health, Epidemiology

\section{Resumo}

Objetivos: determinar a prevalência de anemia ferropriva em escolares e identificar a sensibilidade e a especificidade dos testes laboratoriais, especificamente hemoglobina e hematócrito, no diagnóstico desta deficiência, considerando a ferritina sérica como padrão.

Métodos: o estudo abrangeu 365 alunos no município de Campinas, São Paulo, entre 7 e 14 anos de idade. Foram considerados anêmicos os escolares com valores de hemoglobina abaixo de 11,5 $\mathrm{g} / \mathrm{dl}$ na faixa etária de $5 \leq 8$ anos, 11,9g/dl na faixa etária de $8 \leq 12$ anos; e 12,5g/dl na faixa etária de $12 \leq 15$ anos para o sexo masculino e 11,8g/dl para o feminino. Quanto ao hematócrito, os cortes foram em 34,5\%,35,4\%,37,3\% e $35,7 \%$, respectivamente para as mesmas faixas etárias e sexo. Para a ferritina sérica, $15 \mu \mathrm{g} / \mathrm{dl}$.

Resultados: encontrou-se anemia em 12,4\%, 7,5\% e 19,3\% dos escolares, respectivamente para os valores de hemoglobina, hematócrito e ferritina. A prevalência de anemia ferropriva foi igual a 19,3\%, conforme o padrão ferritina. O teste da hemoglobina apresentou sensibilidade de 12,9\% e especificidade de $87,7 \%$ no diagnóstico da anemia ferropriva, enquanto que o do hematócrito apresentou sensibilidade de $8,6 \%$ e especificidade de $92,8 \%$.

Conclusões: estes valores apontam para o problema do diagnóstico da anemia ferropriva, muitas vezes baseado apenas nos indicadores laboratoriais de baixo custo. É necessário readequar os procedimentos diagnósticos em uso, principalmente na rede básica de saúde.

Palavras-chave Anemia ferropriva, Ferritina, Saúde escolar, Saúde pública, Epidemiologia 


\section{Introdução}

A anemia nutricional tem sido relatada como altamente prevalente em todo o mundo, atingindo as mais diversas faixas etárias $1-5$ e levando à diminuição da atividade cognitiva, comprometimento do desempenho físico e favorecimento da instalação de numerosas infecções. ${ }^{6-8}$

A anemia por deficiência de ferro tem sido identificada como a mais comum das deficiências nutricionais, ocorrendo como resultado de perda sangüínea crônica, perdas urinárias, ingestão e/ou absorção deficiente e aumento do volume sangüíneo. Estimase em 1 bilhão o número de pessoas acometidas pela deficiência de ferro, sendo os grupos mais vulneráveis os lactentes, as crianças e as mulheres.9-13

A deficiência de ferro ocorre quando a quantidade de ferro total do organismo está diminuída, sendo caracterizada pela diminuição da concentração de hemoglobina.14,15 Além da hemoglobina, as medidas do hematócrito 16 e da ferritina têm sido recomendadas no diagnóstico de deficiência de ferro. 17 A alta incidência da deficiência de ferro em todo o planeta, e o fato de terem longa duração ou de serem irreversíveis as conseqüências das deficiências severas de ferro, fizeram com que organizações internacionais como a Organização Mundial da Saúde (OMS), e também organizações de âmbito nacional, estabelecessem como meta principal a erradicação dessa deficiência.

Este estudo teve como objetivo determinar a prevalência de anemia ferropriva em escolares do primeiro grau da rede pública de Campinas, no município de São Paulo, e identificar a sensibilidade e a especificidade dos testes laboratoriais, especificamente da contagem de hemoglobina e do hematócrito, no diagnóstico da anemia ferropriva, considerando a ferritina sérica como padrão.

\section{Métodos}

Trata-se de um estudo transversal, descritivo e analítico desenvolvido no município de Campinas, tendo como população de referência escolares matriculados e freqüentando as escolas estaduais de primeiro grau. As crianças, acompanhadas por seu(s) responsável(is), compareceram às escolas, onde foram submetidas a interrogatório e exame físico, além da coleta de sangue para a análise bioquímica. A participação no estudo foi voluntária, podendo o sujeito desistir a qualquer momento, e sendo o sigilo dos dados garantido pela equipe de pesquisadores. $\mathrm{O}$ estudo teve a aprovação do Comitê de Ética da Pon- tifícia Universidade Católica de Campinas.

Para a seleção dos escolares e para se garantir o espaço amostral ao nível de confiança de $95 \%$ e poder do teste de $80 \%$ foram necessários 245 escolares. Prevendo-se eventuais perdas e sorteando-se $6,5 \%$ de escolares por série, os alunos da primeira à oitava série foram selecionados por região administrativa, de acordo com o número de alunos matriculados, num total de 400. A coleta de dados foi realizada após consentimento por escrito dos responsáveis pelas crianças, contemplando 365 alunos entre 7 e 14 anos de idade, matriculados e freqüentando uma das 6 escolas estudadas - 4 da primeira à oitava série e 2 da quarta à oitava série. Quanto à idade e sexo não houve diferença significativa entre o grupo estudado e o que não compareceu à coleta $(8,75 \%)$.

Cerca de $3 \mathrm{ml}$ de sangue foram colhidos por punção venosa, de cada escolar, após jejum mínimo de 12 horas, em frasco com anticoagulante etilenodiamino tetracético dissódico (EDTA), para as dosagens de hemoglobina e hematócrito, e $3 \mathrm{ml}$ em frasco seco para a dosagem da ferritina. Os frascos foram acondicionados em caixas de isopor contendo gelo reciclável, que foram vedadas e transportadas para análise, num prazo máximo de 2 horas. A dosagem de hemoglobina foi realizada pelo método da cianometahemoglobina, 18 do hematócrito por técnica do microhematócrito 19 e a da ferritina por imuno-ensaio enzimático - ELISA.20

Considerou-se anemia, para hemoglobina, valores abaixo de $11,5 \mathrm{~g} / \mathrm{dl}$ na faixa etária de $5 \leq 8$ anos, $11,9 \mathrm{~g} / \mathrm{d}$ l na faixa etária de $8 \leq 12$ anos e $12,5 \mathrm{~g} / \mathrm{dl}$ na faixa etária de $12 \leq 15$ para o sexo masculino e $11,8 \mathrm{~g} / \mathrm{dl}$ para o feminino, de acordo com a recomendação do Center for Disease Control and Prevention. ${ }^{21}$ Quanto ao hematócrito, os autores sugerem como nível crítico, respectivamente para as mesmas faixas, os cortes em $34,5 \%, 35,4 \%, 37,3 \%$ e $35,7 \%$. Estes valores se referem ao percentil 5 da distribuição de hemoglobina em uma população sadia de acordo com a idade e o sexo. Para a ferritina sérica os mesmos autores indicam ponto de corte em $15 \mu \mathrm{g} / \mathrm{dl}$.

No tratamento estatístico, considerou-se um nível de significância de 5\% ( $\mathrm{p} \leq 0,05)$.

Os dados foram analisados utilizando-se o programa Statistical Package for the Social Sciences. 22

\section{Resultados}

A Tabela 1 mostra os valores hematimétricos encontrados na população estudada. Com relação aos níveis de hemoglobina foi obtida média geral de 12,9 
$\pm 0,9 \mathrm{~g} / \mathrm{dl}$, sendo que a média para as meninas foi de $12,8 \pm 0,9 \mathrm{~g} / \mathrm{dl}$ e para os meninos $13,0 \pm 1,0 \mathrm{~g} / \mathrm{dl}$. O nível médio de hematócrito encontrado foi $39,2 \pm$ $3,2 \%$, sendo que o valor para o sexo feminino foi de $39,0 \pm 3,2 \%$ e para o masculino $39,4 \pm 3,1 \%$. O va- lor médio encontrado para a ferritina foi $34,5 \pm$ $23,3 \mu \mathrm{g} / \mathrm{dl}$. Para as meninas a média foi de $33,8 \pm$ $23,3 \mu \mathrm{g} / \mathrm{dl}$ e para os meninos foi de $35,3 \pm 23,3 \mu \mathrm{g} / \mathrm{dl}$. Não se observou diferença significativa entre os sexos.

Tabela 1

Níveis $(\bar{X} \pm d p)$ de hemoglobina, hematócrito e ferritina dos escolares estudados conforme sexo e idade. Campinas, São Paulo, 1998.

\begin{tabular}{|c|c|c|c|c|c|c|c|}
\hline \multirow{2}{*}{$\begin{array}{l}\text { Idade } \\
\text { (anos) }\end{array}$} & \multirow[b]{2}{*}{$\mathbf{n}$} & \multicolumn{2}{|c|}{ Hemoglobina (g/dl) } & \multicolumn{2}{|c|}{ Hematócrito (\%) } & \multicolumn{2}{|c|}{ Ferritina $(\mu \mathrm{g} / \mathrm{dl})$} \\
\hline & & Feminino & Masculino & Feminino & Masculino & Feminino & Masculino \\
\hline 7 & 38 & $12,4 \pm 0,9$ & $12,5 \pm 1,0$ & $36,6 \pm 6,3$ & $38,2 \pm 2,9$ & $36,2 \pm 24,5$ & $43,5 \pm 36,7$ \\
\hline 8 & 48 & $12,8 \pm 0,7$ & $12,4 \pm 0,9$ & $39,1 \pm 2,2$ & $38,1 \pm 2,7$ & $33,6 \pm 18,9$ & $33,2 \pm 22,7$ \\
\hline 9 & 49 & $12,7 \pm 1,0$ & $12,5 \pm 1,0$ & $38,8 \pm 2,9$ & $37,9 \pm 3,1$ & $36,4 \pm 27,1$ & $36,6 \pm 18,1$ \\
\hline 10 & 49 & $12,6 \pm 0,7$ & $12,9 \pm 0,7$ & $38,6 \pm 2,4$ & $40,2 \pm 2,9$ & $36,6 \pm 26,0$ & $33,5 \pm 17,2$ \\
\hline 11 & 65 & $13,1 \pm 0,8$ & $13,1 \pm 0,8$ & $40,1 \pm 2,5$ & $40,0 \pm 2,5$ & $38,0 \pm 25,4$ & $41,2 \pm 27,9$ \\
\hline 12 & 61 & $12,8 \pm 0,7$ & $13,1 \pm 1,2$ & $39,0 \pm 2,0$ & $40,0 \pm 3,4$ & $32,3 \pm 20,6$ & $34,2 \pm 22,3$ \\
\hline 13 & 34 & $13,2 \pm 1,0$ & $13,4 \pm 1,0$ & $39,9 \pm 2,7$ & $41,0 \pm 2,6$ & $27,6 \pm 17,9$ & $28,1 \pm 18,4$ \\
\hline 14 & 21 & $13,0 \pm 0,9$ & $13,5 \pm 0,7$ & $30,4 \pm 2,6$ & $41,2 \pm 3,0$ & $23,4 \pm 20,3$ & $24,2 \pm 13,3$ \\
\hline Total & 365 & $12,8 \pm 0,9$ & $13,0 \pm 1,0$ & $39,0 \pm 3,2$ & $39,4 \pm 3,1$ & $33,8 \pm 23,3$ & $35,3 \pm 23,3$ \\
\hline
\end{tabular}

A análise dos valores de hemoglobina, hematócrito e ferritina (Tabela 2) mostrou, respectivamente, uma prevalência de anemia de $12,4 \%$ (45 casos), 7,5\% (27 casos) e 19,3\% (70 casos) na população estudada. Considerando-se, neste estudo, a ferritina como o melhor indicador para a anemia ferropriva, verifica-se que o teste da hemoglobina apresentou sensibilidade de $12,9 \%$ e especificidade de $87,7 \%$, enquanto que o do hematócrito apresentou sensibilidade de $8,6 \%$ e especificidade de $92,8 \%$ na determinação da anemia ferropriva, isto é, ambos deixam de identificar cerca de $90 \%$ das crianças com anemia, embora considerem com anemia apenas cerca de $10 \%$ das crianças sem deficiência de ferro.

A anemia ferropriva, medida pela ferritina sérica, atingiu igualmente os dois sexos, com prevalência de $19,3 \%$, sendo $18,8 \%$ entre o sexo feminino e $20,0 \%$ entre o masculino.

Tabela 2

Sensibilidade e especificidade dos testes de hemoglobina e hematócrito na determinação da anemia ferropriva em escolares tendo como padrão a feritina sérica.

\begin{tabular}{lcccr}
\hline & \multicolumn{4}{c}{ Ferritina $<15 \mu \mathrm{g} / \mathrm{dl}$} \\
\cline { 2 - 5 } & Anemia & \multicolumn{1}{c}{ Sim } & Não & Total \\
\hline Hemoglogina & Sim & $9(12,9 \%) \mathrm{s}$ & $36(12,3 \%)$ & 45 \\
& Não & $61(87,1 \%)$ & $256(87,7 \%)$ e & 317 \\
Hematócrito & Sim & $6(8,6 \%) \mathrm{s}$ & $21(7,2 \%)$ & 27 \\
Total & Não & $64(91,4 \%)$ & $271(92,8 \%)$ e & 335 \\
& - & $70(100,0 \%)$ & $292(100,0 \%)$ & 362 \\
\hline
\end{tabular}

$\mathrm{s}=$ sensibilidade $; \mathrm{e}=$ especificidade 


\section{Discussão}

A discrepância quanto aos valores obtidos para a anemia pelos diferentes testes pode ser explicada pelos diferentes estágios da deficiência de ferro no organismo. A deficiência de ferro ocorre quando a quantidade de ferro total do organismo está diminuída. Três estágios podem ser identificados, cada um mudando gradativamente para o outro de acordo com a gravidade da deficiência. O primeiro se caracteriza pela diminuição dos estoques de ferro, refletida pela queda da ferritina sérica. No segundo estágio, a saturação da transferrina está diminuída. No terceiro estágio, as células vermelhas são microcíticas e hipocrômicas e os níveis de hemoglobina estão reduzidos. $14,15,17$

Segundo Herbert, 17 o nível de hemoglobina não é baixo nem na depleção de ferro nem na eritropoiese deficiente de ferro; por isso outros métodos devem ser usados para determinar esses dois primeiros estágios da deficiência de ferro, sendo a dosagem da ferritina sérica um dos mais indicados.

Os valores obtidos para sensibilidade e especificidade dos testes de hemoglobina e hematócrito, comparativamente à ferritina, apontam para o problema do diagnóstico da anemia ferropriva, muitas vezes baseado apenas nos indicadores laboratoriais de baixo custo como é o caso da contagem da hemoglobina e do hematócrito. Considerando que o combate à anemia ferropriva representa hoje uma das prioridades para os profissionais de saúde, devido à sua elevada prevalência e às suas conseqüências no desenvolvimento e na mortalidade infantil, principalmente entre os menores de 2 anos de idade, é necessário reavaliar os procedimentos diagnósticos em uso, principalmente na rede básica de saúde.

\section{Agradecimentos}

Órgãos financiadores: Fundação de Apoio à Pesquisa do Estado de São Paulo (FAPESP), Conselho Nacional de Desenvolvimento Científico e Tecnológico (CNPq), Coordenadoria de Ensino e Apoio à Pesquisa da Universidade Católica de Campinas, São Paulo (CEAP/PUC, Campinas, SP). 


\section{Referências}

1. Kahn R, Romslo I, Lamvik J. Anemia in general practice. Scand J Clin Lab Invest 1990; 200S: 41-5.

2. Romani AS, Lira PI, Batista Filho M, Sequeira LA, Freitas CL. Anemias em pré-escolares: diagnóstico, tratamento e avaliação, Recife - PE, Brasil. Arch Latinoam Nutr 1991; 41: 159-67.

3. Bushnell FKL. A guide to primary care of iron-deficiency anemia. Nurse Pract 1992; 17: 68-74.

4. Cook JD, Skikne BS, Baynes RD. Iron deficiency: the global perspective. Adv Exp Med Biol 1994; 356: 219-28.

5. Batista Filho MB, Ferreira LOC. Prevenção e tratamento da anemia nutricional ferropriva: novos enfoques e perspectivas. Cad Saúde Pública 1996; 12: 411-5

6. Gutiérrez-Sigler D, Colomer-Revuelta J, Barona C, Momparler P, Colomer-Revuelta C. Asociación entre carencia de hierro y transtornos del aprendizaje en la edad preescolar. Gac Sanit 1992; 6: 207-11.

7. Thaver IH, Baig L, Haq IU, Iqbal R. Anemia in children: part II. Should primary health care providers prescribe iron supplements by the observation and presence of assumed symptons? J Pak Med Assoc 1994; 4: 284-5.

8. Leshan L, Gottlieb M, Mark D. Anemia is prevalent in an urban African-American adolescent population. Arch Fam Med 1995; 4: 433-7.

9. Lönnerdal B, Dewey KG. Epidemiologia da deficiência de ferro no lactente e na criança. An Nestlé 1996; 52: 11-7.

10. Takahashi H, Yang SI, Ueda Y, Kim M, Yamamoto, T. Influence of intact and partially hydrolysed guar gum on iron utilization in rats fed on iron-deficient diets. Comp Biochem Physiol 1994; 109A: 75-82.

11. Trowbridge FL, Harris SS, Cook J, Dunn JT, Florentino RF, Kodyat BA, Mannar MGV, Reddy V, Tontisirin K, Underwood BA, Yip R. Coordinated strategies for controlling micronutrient malnutrition: a technical workshop. J Nutr 1993; 123: 775-87.
12. Yip R. Iron deficiency contemporary scientific issues and international programmatic approaches. J Nutr 1994; 124: 1479S-90S

13. Freire WB. La anemia por deficiencia de hierro: estrategias de la OPS/OMS para combatirla. Salud Public 1998; 40: 199-205.

14. Hallberg L, Bengtsson C, Lapidus L, Lindstedt G, Lundberg PA, Hultén L. Screening for iron deficiency: an analysis based on bone-marrow examinations and serum ferritin determinations in a population sample of women. Br J Haematol 1993; 85: 787-98.

15. Roodenburg AJC. Iron suplementation during pregnancy. Eur J Obstet Gynecol Reprod Biol 1995; 61: 65-71.

16. Zein ZA. Haematocrit levels and anaemia in Ethiopian children. East Afr Med J 1991; 68: 412-9.

17. Herbert V. Recommended dietary intakes (RDI) of iron in humans. Am J Clin Nutr 1987; 45: 679-86.

18. Drabkin DL, Austin SH. Spectrophotometric studies. II. Preparation from washed blood cells, nitride oxide hemoglobin and sulphemoglobin. J Biol Chem 1935; 112: 51-65.

19. Haden RI. Principles of hematology. Philadelphia: Lea \& Febiger; 1946

20. Tietz NW. Fundamentals of clinical chemistry. 3. ed. Philadelphia: Saunders; 1987.

21. Yip R, Parvanta I, Cogswell SM, McDonnell SM, Bowman BA, Grummer-Strawn LM, Towbridge FL, Gunter EW, Looker AC, Van-Assendelf OW, Bakes-Martin RC, Bern C, Clark LD, Perry GS, Scanlon KS, Sherry B, Zyrkowski CL. Recommendations to prevent and control iron deficiency in the United States. Morb Mortal Wkly Rep 1998; 47: 1-29.

22. Statistical Package for the Social Sciences, SPSS for Windows. Advanced statistics: release 5 [computer program]. Chicago: SPSS; 1992. 